\title{
Too much sitting and too little exercise: sedentary behavior and health
}

\author{
Muito tempo sentado e pouca atividade física: comportamento sedentário e saúde
}

\begin{tabular}{l} 
AUTHOR'S \\
\hline Neville Owen' ${ }^{1}$ D \\
1 Baker Heart and Diabetes Institute and \\
Swinburne University of Technology, Melbourne \\
Australia. \\
CORRESPONDING \\
Neville Owen \\
neville.owen@baker.edu.au \\
Baker Heart and Diabetes Institute \\
Level 4, 99 Commercial Road, \\
Melbourne Vic 3004 Australia \\
PO Box 6492, Melbourne, Vic 3004 \\
Australia \\
DOI
\end{tabular}

$10.12820 /$ rbafs.23e0001

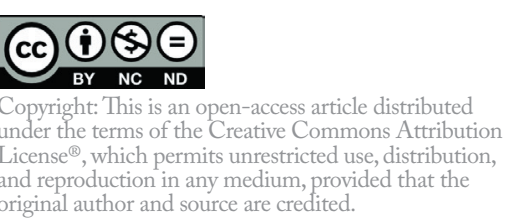

\section{Introduction}

Sedentary behaviors are characterized by a seated posture and low energy expenditure, and are ubiquitous in developed and developing countries ${ }^{1}$. Too much sitting - along with lack of exercise and too little physical activity - is now understood to increase the risk of major chronic diseases, and can result in functional disability and reduced quality of life ${ }^{2}$.

The sedentary behavior concept, and the rapidly expanding body of evidence on its health consequences in no way contradict established recommendations on moderate-vigorous physical activity and health. Rather, the focus on sedentary behavior expands options for increasing daily energy expenditure, enhancing related and unique healthful biological processes and identifying more precisely those groups in society whose health is most at risk through inactivity ${ }^{3}$.

This series of papers on Sedentary Behavior of the Brazilian Journal of Physical Activity and Health provide important new points of reference, not only for the physical activity and health and chronic disease prevention field in Brazil, but also for other Latin American countries and more broadly across the globe. These papers speak for themselves, in terms of the quality of the research reported here and the relevance for population health. They cover a broad age spectrum, with novel evidence relating to children, adolescents, the general adult population and older people. Such evidence is important for physical activity and population health internationally, given the emerging body of work that shows the unique factors that can influence chronic disease risk through physical inactivity, depending on cultural, environmental, climatic and other relevant attributes that vary by region and country ${ }^{4}$.

While these are a set of papers from a particular country, they are nicely representative of the development of research in the field more broadly, with a predominance of descriptive-epidemiology studies (with four papers on the correlates of sedentary behavior; three papers on health-related correlates; and, one paper on objective measurement of sedentary behavior in adolescents). One paper in the series reports findings of an intervention trial to change screen time in adolescents. Such a balance of papers at this stage of the development of the science of sedentary behaviour and health is appropriate - mapping the relevant behavioral and contextual territory, and building the base of understanding on measurement provides a solid set of underpinnings for the field. In the future, intervention trials will be able to be argued for and developed with reference to the insights provided by this knowledge. 
In addressing health consequences, social and environmental determinants of sedentary behavior, and intervention effects, this set of papers also covers key elements of the broader research spectrum that are needed to fully make sense of an important emerging public-health topic. Much of the evidence on sedentary behavior and health has to this point come from cross-sectional and prospective epidemiological observational studies, up until recently reporting findings based on self-report measures of sedentary behavior. ${ }^{2}$ More recently, we have begun to see prospective epidemiological studies with objective measurement of sedentary behavior and physical activity $^{5}$, which will further reinforce the scientific and public-health importance of previous research findings.

The sedentary behavior and health research agenda for the future should emphasize evidence from studies involving a broadly-based and rigorous analysis of evidence on the determinants of sedentary behavior, which can then inform intervention studies that help us to derive evidence of a more-strongly causal nature on the feasibility and benefits of changing sedentary behavior ${ }^{6}$.

Many new opportunities for public health intervention exist. As we have developed our sedentary behavior and health research program in Australia over the past 10 years, we have used two main sets of guiding ideas: the Behavioral Epidemiology framework ${ }_{;}^{7}$ and, an Ecological Model of sedentary behavior ${ }^{6,8}$.

\section{Sedentary behavior and health outcomes: building systematically on observational- study evidence}

To guide a comprehensive and strategic approach to research on sedentary behavior and health, we have applied the Behavioral Epidemiology Framework. Sallis and Owen put forward this framework in our 1999 book - Physical Activity and Behavioral Medicine. ${ }^{7}$ In summary, behavioral epidemiology in this context identifies six phases of research ${ }^{1}$ :

- identifying relationships of sedentary behavior with health outcomes;

- measuring sedentary behaviour;

- characterizing prevalence and variations of sedentary behavior in populations;

- identifying the determinants of sedentary behaviour;

- developing and testing interventions to influence sedentary behaviour;

- using the relevant evidence to inform public health guidelines and policy.
In our program in Australia, we have been placing a high priority on the first phase of the Behavioural Epidemiology Framework (identifying relationships of sedentary behavior with health outcomes). Epidemiological evidence - both inferential and descriptive - plays a central role, but in itself is insufficient to provide important elements of the relevant evidence base. ${ }^{2,3}$ This is particularly so in the context of understanding the mechanisms by which sedentary behavior can have adverse impacts on health outcomes. This element of the agenda requires experimental evidence that brings the logic of sedentary behavior research closer to being able to make unambiguous causal inferences ${ }^{3}$. It also can build important interdisciplinary links - particularly to underlying disciplines in exercise science and integrative biology.

Evidence from epidemiological observational studies showing cross-sectional and prospective relationships of sedentary behavior with indices of health and health outcomes can be particularly helpful in generating novel hypotheses. For example, in studies with Japanese older adults, we have identified different cross-sectional associations of sedentary behaviors with health-related indices. Passive sedentary behaviors (for example television viewing time) were found to have adverse associations with indices of wellbeing; in contrast, mentally-active sedentary behaviors (such as reading and computer use) had beneficial associations ${ }^{9}$.

Several key scientific questions arise for the sedentary behavior and health field, in relation to the potential protective role of moderate-vigorous physical activity as a counter to the adverse effects of time spent sitting. Strong evidence in this regard has recently come from our meta-synthesis of data from multiple prospective observational studies, showing that the adverse consequences for all-cause mortality risk of overall sitting time and television viewing time can be partly attenuated by moderate-vigorous physical activity $^{10}$. However, those relationships were only apparent for very high volumes of activity - levels of physical activity participation that are likely to be unrealistic for current cohorts of urban-dwelling adults ${ }^{11}$. A limitation of such epidemiological evidence to this point, has been the reliance on imprecise self-report measures of physical activity and sedentary behavior. In the near future, several studies will build on prospective epidemiologic-observational evidence, where the relevant exposures are measured objectively using accelerometers ${ }^{5}$.

Our program of disciplinary-driven experimental enquiry - identifying potential mechanistic under- 
pinnings for the relationships of sedentary behavior with health outcomes - has been pursued at the Baker Heart and Diabetes Institute over the past 10 years, particularly building on a compelling set of findings from Genevieve Healy's body of observational study evidence on the relationships of interrupting sedentary time with cardiometabolic risk biomarkers. ${ }^{12}$ We have subsequently shown that interrupting prolonged sitting can have acute beneficial effects on blood glucose and insulin levels, ${ }^{13}$ and other biomarkers in ways that are both related to and distinct from those associated with moderate-vigorous physical activity ${ }^{14}$.

\section{Sedentary behavior determinants and interventions}

Conceptually, we have continued to develop an ecological model of sedentary behavior, focusing on multiple levels of factors that can act as determinants of sedentary behaviors in particular contexts - behavior settings. These include the workplace, the transportation context (particularly in the use of private automobiles), the domestic environment and a range of community settings. In this conceptual model, evidence on associations of environmental, social and personal-level attributes with sedentary behavior can provide helpful evidence to inform the development of settings based interventions ${ }^{6,8,15}$.

Promising findings are emerging from controlled trials in workplaces. In a cluster-randomized trial, Healy and colleagues examined the effects on objectively-assessed sedentary behavior patterns and cardiometabolic risk biomarkers of a multicomponent intervention that included the provision of sit-stand workstations. Significant reductions in workplace sitting time resulted, with those changes largely attributable to sitting time being replaced by time spent standing rather than walking ${ }^{16}$. Significant improvements in some cardiometabolic risk biomarkers were identified among those who made the greatest behavioral changes, particularly via more stepping ${ }^{17}$.

Workplace trials of this nature provide a model that could readily be adapted to the school setting. A combination of environmental opportunity - the provision of sit-to-stand desks - with education and information for students and families and teacher training, potentially could be highly effective in reducing sitting time in the educational context for children and adolescents.

In the context of sedentary behaviour intervention trials, it would be remiss not to emphasize the fundamental importance of high-quality measurement. For sedentary behavior, device-based objective measurement methods will be key to the future development of the science (as the paper by Ramos and colleagues in this issue illustrates). However, for the evaluation of interventions, linking behavioral measurement to environmental context is crucial. For this purpose, there remains the need to employ a combination of device-derived and self-report measures. In order to characterize the context of sedentary behaviors in ways that will inform the development of setting-specific interventions (including the workplace, transportation and the domestic environment) and evaluate the relevant behavioral and health-related changes, self-report will remain an important element of the measurement dimensions of sedentary behavior science, for the foreseeable future ${ }^{18}$.

The following series of papers report unique findings that provide key building blocks for subsequent studies on sedentary behaviour and health. All deserve thorough reading. They illustrate resourceful and well-grounded scientific approaches to an already well-established public health issue for Brazil and other Latin-American countries. Over the next several years, they will lead to many other studies by new and emerging Brazilian research investigators and their colleagues.

\section{References}

1. Owen N, Healy GN, Matthews CE, Dunstan DW. Too much sitting: The population health science of sedentary behavior. Exerc Sport Sci Rev. 2010;38(3):105-13.

2. Owen N. Chapter 1: Emergence of research on sedentary behaviour and health. In Zhu W, Owen N, (Eds). Sedentary behavior and health concepts, assessments, and interventions. pp. 3-12, 355-358. Champaign, IL: Human Kinetics. 2017 (http://www.humankinetics.com/products/all-products/ Sedentary-Behavior-and-Health)

3. Hadgraft N, Owen N. Sedentary behavior and health: broadening the knowledge base and strengthening the science. Res QExerc Sport. 2017;88(2):123-29.

4. Mabry R, Koohsari MJ, Bull F, Owen N. A systematic review of physical activity and sedentary behaviour in the oilproducing countries of the Arabian Peninsula. BMC Public Health. 2016;16:1003.

5. Diaz KM, Howard VJ, Hutto B, Colabianchi N, Vena JE, Safford MM, et al. Patterns of Sedentary Behavior and Mortality in U.S.Middle-Aged and Older Adults: A National Cohort Study. Ann Intern Med. 2017;167(7):465-75.

6. Owen N, Sugiyama T, Eakin EE, Gardiner PA, Tremblay MS, Sallis JF. Adults' sedentary behavior: Determinants and interventions. Am J Prev Med. 2011;41(2):189-96.

7. Sallis JF, Owen N. Physical Activity and Behavioral Medicine. Thousand Oaks, CA: Sage; 1999. Japanese-Language Edition published in 2000 by Kitaohji Shobo, Tokyo.

8. Sallis JF, Owen N. Ecological models of health behavior. In: Glanz K, Rimer BK, Viswanath K, (Eds). Health Behavior Theory Research and Practice 5th Edn. San Francisco: Jossey-Bass; 2015. p. 43-64. 
9. Kikuchi H, Inoue S, Sugiyama T, Owen N, Oka K, Nakaya $\mathrm{T}$, et al. Distinct associations of different sedentary behaviors with health-related attributes among older adults. Prev Med. 2014;67:335-9.

10. Ekelund U, Steene-Johannessen J, Brown WJ, Wang Fagerland M, Owen N, Powell KE, et al. Does physical activity attenuate, or even eliminate the detrimental association of sitting time with mortality? A harmonised meta-analysis of data from more than one million men and women. Lancet. 2016;388(10051):1302-10.

11. Sparling PB, Howard BJ, Dunstan DW, Owen N. Recommendations for physical activity in older adults. BMJ. 2015;350:h100.

12. Healy GN, Winkler EAH, Owen N, Anuradha S, Dunstan DW. Replacing sitting time with standing or stepping: associations with cardio-metabolic risk biomarkers. Eur Heart J. 2015;36(39):2643-49.

13. Dempsey PC, Larsen RN, Sethi P, Sacre JW, Straznicky NE, Cohen ND, et al. Benefits for Type 2 diabetes of interrupting prolonged sitting with brief bouts of light walking or simple resistance exercises. Diabetes Care. 2016;39(6):964-72.

14. Grace M, Dempsey P, Sethi P, Mundra P, Mellett N, Weir J, et al. Breaking up prolonged sitting alters the postprandial plasma lipidomic profile of adults with Type 2 Diabetes. J Clin Endocrinol Metab. 2017;102(6):1991-9.
15. Koohsari MJ, Sugiyama T, Sahlqvist S, Mavoa S, Hadgraft N, Owen N. Neighborhood environmental attributes and adults' sedentary behaviors: Review and research agenda. Prev Med. 2015;77:141-49.

16. Healy GN, Eakin EG, Owen N, LaMontagne AD, Moodie $\mathrm{M}$, Winkler EAH, et al. A cluster RCT to reduce office workers' sitting time: impact on activity outcomes. Med Sci Sports Exerc. 2016;48(9):1787-97.

17. Winkler E, Chastin S, Eakin EG, Owen N, LaMontagne AD, Moodie M, Dempsey PD, Kingwell B, Dunstan DW, Healy GN. Cardio-metabolic impact of changing sitting, standing, and stepping in the workplace. Med Sci Sports Exerc. 2018;50(3):516-24.

18. Clark B, Lynch BM, Winkler E, Gardiner PA, Healy G, Dunstan DW, et al. Validity of a multi-context sitting questionnaire across demographically diverse population groups: AusDiab3. Int J Behav Nutr Phys Act. 2015;12(148).

Recebido: 05/07/2017

Aprovado: 12/12/2017 